Egyptian

Orthodontic Journal

\title{
STUDY OF CRANIOFACIAL MORPHOLOGY IN CHILDREN WITH JUVENILE DIABETES
}

\author{
ABDULLAH AL MUSHAYT* \\ ABEER AL-NWAISER ${ }^{* *}$
}

\section{ABSTRACT}

Previous studies have shown that patients with Juvenile Diabetes have growth retardation. Also, previous researches have also that there is relationship between the general body growth and craniofacial maturation. The purpose of this study was to evaluate the craniofacial morphology and to relate it to the degree of diabetes in children with Juvenile Diabetes. Thirty growing children (age range 14-16 year) who had Juvenile Diabetes (JD) for 3-5 years and that were been treated by daily administration with insulin were studied. Another healthy thirty children with no familial history of any endocrinal disorders or Juvenile Diabetes and who attend the orthodontic/Pedodontic clinics of KAAV seeking orthodontic treatment were chosen. Lateral cephalomteric radiographs were taken for each patient after consenting patients to participate in this study. All cephalometric radiographs were corrected for magnification distortion and analyzed to evaluate their craniofacial growth status. The results showed that ID patients are underdeveloped in most of the craniofacial skeletal and soft tissue parameters when compared with the control group.

\section{INTRODUCTION}

Diabetes mellitus (DM) is described as a clinical syndrome characterized by inappropriately elevated fasting and /or post-prandial blood glucose, and by the development of long-term microvascular, macrovascular and neuropathic

\footnotetext{
* BDS, Pedicert. , PhD: Assistant Professor of Pediatric Dentistry and Chairman, OBSC Department, KAAU, KSA.

** BDS, MS, PhD: Assistant Professor of Pediatric Dentistry, Preventive Dental Sciences Department, KAAU, KSA.
} 
changes. ${ }^{1}$ It is a disturbance of energy metabolism due to deficiency of insulin or its action. It is characterized by altered homeostasis of carbohydrates, proteins and fats. ${ }^{2}$

Insulin-dependent diabetes mellitus (IDDM) is the most frequent endocrinal metabolic disorder of childhood, and is characterized by severe insulinopenia, requiring exogenous insulin to prevent ketosis and to preserve life. ${ }^{2}$ According to the National Diabetes Data Group (1979) ${ }^{3}$, the typical manifestations IDDM are glucosuria, ketonuria, and random PG (Plasma Glucose) $>200$. Juvenile diabetics are defined as patients in whom the onset of the disease starts before the age of 15 years. ${ }^{4}$ In these patients, the disease is called juvenile diabetes (JD), Ketosis prone diabetes, or brittle diabetes. ${ }^{5}$

The occurrence of the disease in the United States is reported as $1.42 / 1000 ;{ }^{6} 1.9 / 1000$ among school age children with an estimated ratio of $0.7 / 1000$ at 5 years of age to $2.8 / 1000$ at 16 years. ${ }^{2}$ Males are more frequently diagnosed with the disease in early childhood, while females are more frequently diagnosed in late childhood. ${ }^{7}$ The overall prevalence of diabetes mellitus in Saudi Arabia was reported to be $9.7 \%$ and $7.0 \%$, obesity $13.05 \%$ and $20.26 \%$, overweight $27.23 \%$ and $25.20 \%$, and hypertension $5.39 \%$ and $3.65 \%$ in the adult male and female populations respectively. ${ }^{8}$ In Egypt, the prevalence was estimated to be $0.33 / 1000$ for females and 2/1000 for males. ${ }^{9}$ Patients with IDDM are subject to ketosis and are sensitive to insulin. Physical manifestations are absent in mild diabetes. In severe neglected IDDM, there is dehydration, ketosis, dry skin and mucous membrane, and hypotension. There may also be skin infections, peripheral neuropathy, peripheral vascular insufficiency, cataract and diminished visual power from retinopathy. ${ }^{5}$ Complications of Juvenile IDDM include osteolysis (diabetic osteopathy), characterized by juxta-articular bone defects occurring in the feet, which may be self-healing. ${ }^{10}$ There is increased glycosylation of skin collagen in IDDM due to increased accumulation of stiff and less degradable collagen. Also, limited joint mobility is reported in $92 \%$ of patients with IDDM between the ages of 10-20 years. It is postulated that IDDM is associated with growth retardation. ${ }^{11,12}$ Hand-wrist radiographs have been studied in juvenile diabetics. ${ }^{13}$ There is delayed appearance or delayed development of a center of ossification, usually of a carpal bone. These defects occur twice as frequently in boys than in girls, and the total incidence of juvenile diabetics with anomalies and developmental defects is $24.3 \%$. There is also retardation of bone growth in $60 \%$ of diabetic males and $51 \%$ of diabetic females. The longer the duration of diabetes, the greater the tendency to bone growth retardation. The decreased bone mass in diabetics has been explained by 
decreased proliferative capacity of the diabetic fibroblasts, and early senescence of all cells has been suggested as basic to the diabetic problem. This degeneration would lead to early osteopenia in bone. ${ }^{14}$ The yearly bone loss was reported to be $1.35 \%$ in patients with $\mathrm{JD} .^{15}$ In addition, the rate of bone mineral loss is significantly greater among patients with a deterioration of the metabolic state, despite increasing insulin dosage, when compared to patients with unchanged or improved insulin secretion. This may indicate that the exogenous insulin administration doesn't fully compensate for the decrease in endogenous insulin secretion. These studies also showed increased bone resorption in JD patients with no signs of vitamin D deficiency associated with the disease. Vertebral bone density has been studied in IDDM children. ${ }^{16}$ It was found that diabetic children exhibited cortical bone density that was slightly, but significantly, lower than the controls. The decrease in cortical bone density in the diabetic group did not correlate with age, sex, duration of diabetes, or glycosylated hemoglobin levels. These results suggested that in children with uncomplicated IDDM, decreased vertebral bone density is a minor abnormality that affects only cortical bone. El-Bialy, et al., 2000 studied the craniofacial morphology and skeletal maturation in juvenile diabetics. ${ }^{17}$ They concluded that the diabetic patients showed decreased skeletal maturation and decreased cephalometric linear and angular measurements when compared with the control group. However, there was no other reported data in Saudi Arabia that investigated this deficient craniofacial skeletal growth in JD patients. The aim of the study was to study the craniofacial morphology of JD.

\section{Material and Methods}

The experimental group consisted of 30 patients with juvenile diabetes chosen from the Department of Pediatrics at King Abdul Aziz University hospital, Jeddah, KSA. The selection criteria were:

1.Patient ages between $14-16$ years

2. Onset of diabetes was at least 5-6 years before enrollment in the study

3. No display of any other systemic or local diseases that could affect the general body development or craniofacial morphology. The control group consisted of 30 nondiabetic normal individuals in the same age range (14-16 years) and paired with the JD patients. They were elected from the outpatient clinic of the Faculty of Dentistry, King Abdul Aziz University hospital, Jeddah, KSA. Selected patients were seeking routine dental treatment other than orthodontics 
and were without recognized abnormalities. For each subject enrolled in this study, the following records were taken:

1- Lateral cephalometric radiograph with the teeth in habitual occlusion; cephalometric landmarks and lines were identified (Figure1)

2- Detailed medical and dental histories.

All cephalometric tracings were corrected for magnification distortion. In order to minimize the error of measurements, two investigators working independently performed all linear and angular measurements. When their measurements were within $0.5 \mathrm{~mm}$ for the linear measurements, 0.5 degrees for the angular measurements, the average of the two measurements was used. If there was a greater disparity, each investigator performed two additional measurements and either the mean of the four measurements, or if there was an obvious misreading, the mean of the homogenous three measurements was used.

To compare the samples, differences among the means were established using the student $t$-test which evaluated the difference in all parameters. The association between the craniofacial variables and those describing skeletal maturation were assessed by means of correlation coefficient test.

\section{RESULTS}

Figure 1 show templates of the cephalometric analyses for both JD and control groups.

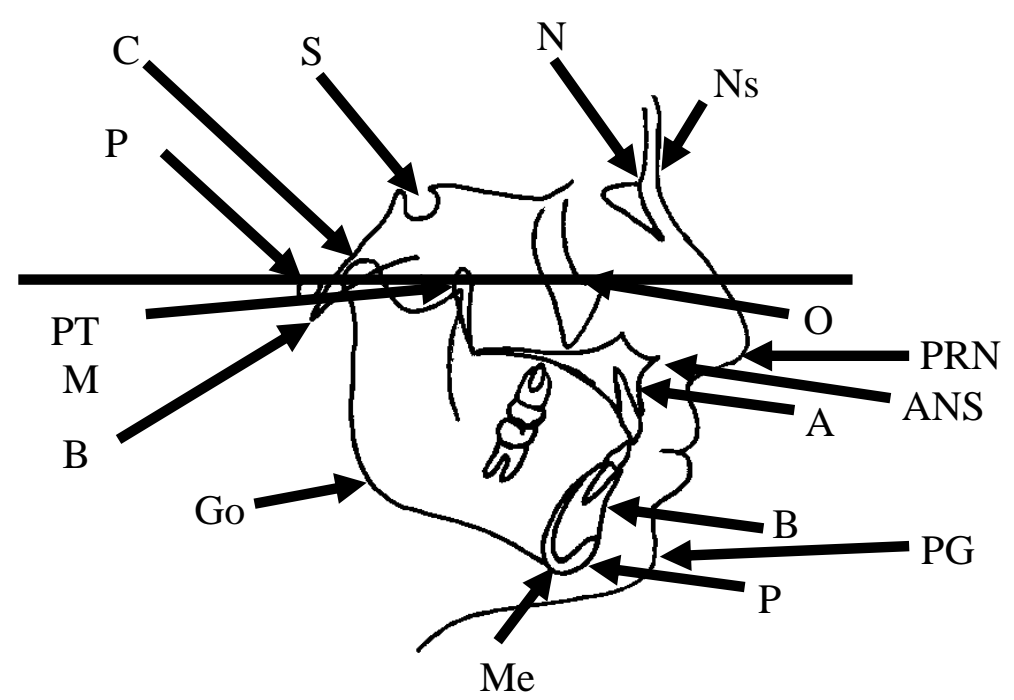

Volume 28 - December 2005 
Egyptian

Orthodontic Journal

Figure 1: Cephalometric landmarks

Table 1. Comparison of skeletal cephalometric measurements for juvenile diabetic patients (S) and control group (C).

Variables

Linear measurements

S-N
S-Ba
S-Go
S-Ar
S-GN
A-PM
CD-Go
GO-Me
N-Me
N-ANS
ANS-Me
(N-Me) $\%$
-Me)(N-Me) $\%$

Angular measurements

$\begin{array}{cccccc}\text { S-N-A } & 82.3 & 3.5 & 84.1 & 2.6 & <0.05 \\ \text { S-N-B } & 77.2 & 3.1 & 78.6 & 2.9 & >0.05 \\ \text { A-N-B } & 5.3 & 2.2 & 5.7 & 2.8 & >0.01 \\ \text { S-N-Pg } & 78.4 & 2.7 & 78.7 & 3.2 & >0.05 \\ \text { Facial angle } & 86.6 & 2.9 & 87.3 & 3.3 & >0.05 \\ \text { Cranial base angle } & 124.7 & 3.8 & 129.2 & 2.9 & <0.05 \\ \text { N-S-Ar } & 117.4 & 5.3 & 124.3 & 4.3 & <0.001 \\ \text { Gonial angle } & 113.4 & 4.3 & 115.6 & 3.9 & >0.05 \\ \text { SN-mand. PL. } & 34.4 & 5.5 & 33.7 & 4.7 & >0.05 \\ \text { SN-Palatal PL. } & 9.2 & 2.8 & 12.6 & 3.4 & <0.01 \\ \text { FH-mand.PL } & 27.3 & 3.8 & 26.2 & 4.2 & >0.05 \\ \text { Y-Axis } & 59.5 & 3.7 & 62.7 & 2.5 & <0.01 \\ \text { Pm-S-Ba } & 56.5 & 4.8 & 57.5 & 4.5 & >0.05\end{array}$

Table 1 shows comparison of skeletal linear and angular measurements between JD and control groups. Table 2 shows comparison of soft tissue linear

Volume 28 - December 2005

\begin{tabular}{|c|c|c|c|c|}
\hline \multicolumn{2}{|c|}{$\mathbf{S}$} & \multicolumn{2}{|c|}{ C } & \multirow{2}{*}{$P$} \\
\hline $\mathbf{X}$ & SD & $\mathbf{X}$ & SD & \\
\hline 70.5 & 3.2 & 73 & 3.1 & $<0.05$ \\
\hline 45.3 & 5.3 & 48.7 & 3.2 & $<0.05$ \\
\hline 72.3 & 4.1 & 77.4 & 4.8 & $<0.001$ \\
\hline 32.1 & 2.9 & 35.1 & 2.8 & $<0.001$ \\
\hline 124.2 & 5.9 & 128.7 & 5.2 & $<0.01$ \\
\hline 47.8 & 4.3 & 53.1 & 2.3 & $<0.001$ \\
\hline 50.2 & 3.1 & 56.3 & 2.9 & $<0.001$ \\
\hline 71.8 & 5.4 & 76.8 & 2.9 & $<0.01$ \\
\hline 115.7 & 5.8 & 124.8 & 6.2 & $<0.001$ \\
\hline 51.7 & 3.2 & 56.8 & 3.5 & $<0.001$ \\
\hline 64.9 & 3.9 & 69.6 & 4.6 & $<0.01$ \\
\hline 60.5 & 3.5 & 64.4 & 3.2 & $<0.05$ \\
\hline 54.1 & 3.1 & 55.2 & 2.9 & $>0.05$ \\
\hline
\end{tabular}


and angular measurements between JD and control groups. It can be seen that JD patients are below average of all skeletal and sift tissue parameters than the control group.

Table 2. Comparison of soft tissue cephalometric measurements for JD patients (S), and control group (C).

Variables

Linear measurements

$\begin{array}{lccccc}\text { N-PRN } & 47.8 & 5.6 & 54.2 & 4.4 & <0.001 \\ \text { PRN__ N-PGs. } & 21.6 & 3.2 & 26.7 & 2.5 & <0.001 \\ \text { PG-PGs } & 13.2 & 2.1 & 13.8 & 2.3 & >0.05 \\ \text { Ricketts UL } & -2.1 & 1.8 & -1.5 & 1.8 & >0.05 \\ \text { Ricketts LL } & 1.5 & 1.3 & 2.2 & 1.9 & >0.05 \\ \text { Angular measurements } & & & & & \\ \text { S-N-PRN } & 117.3 & 4.6 & 113.2 & 4.2 & <0.05 \\ \text { N-PRN-PG } & 136.5 & 5.3 & 130.6 & 5.7 & <0.01\end{array}$

\section{DISCUSSION}

Juvenile diabetes mellitus has been shown to affect the general growth of patients with earlier onset of the disease, especially onset before or around the circumpubertal growth spurts. ${ }^{13,14,18}$ Many investigators have reported that this growth impairment in JD patients is more significant in boys than in girls. ${ }^{19,20}$ Males comprised the selected sample in order to obtain more representative data regarding skeletal maturation from hand-wrist radiographs. To see the effect of diabetes on the circumpubertal growth spurt, the selected sample ages ranged between 14 and 16 years. These patients had been diagnosed with diabetes at least 5 years before the study. The lateral cephalometric analysis showed that all linear measurements were decreased in JD patients. These linear measurements have been reported by many investigators as being craniofacial growth indicators. ${ }^{21,22}$ Study of the cephalometric angular measurements in JD patients revealed that there was decreased ANB angle, which may be due to a decreased SNA angle. This may indicate that JD may affect the forward growth of the maxilla. In addition, S-N-Ar angle was reported to increase during successive years of normal growth. JD patients showed a decreased S-N-Pg angle. Although this decrease is not significant, it may indicate that JD can affect chin prognathism, which was reported by Kerr23 to increase significantly between 5 
and 15 years. Gonial angle showed slight but not significant increase in juvenile diabetic patients. This angle was reported by Broadbent 21 to decrease during the successive years of normal growth of the mandible. The mandibular plane inclination to the anterior cranial base angle was increased, but not significantly in JD patients. This angle was reported by Broadbent ${ }^{21}$ and $\mathrm{Kerr}^{23}$ to decrease between 5 and 15 years in normal individuals. The inclination of the maxilla to the anterior cranial base (SN-palatal plane) showed a significant decrease in JD patients. This angle was also reported by Broadbent and Kerr to increase between 5 and 15 years in normal individuals. The inclination of the body of the mandible to the Frankfort horizontal plane shows slight but not significant increase in JD patients. This angle has been reported to show a slight decrease in a normal sample during successive years of growth up to puberty. The Y-axis, the downward and forward mandibular growth indicator, is significantly decreased in JD patients. The bony nasopharynx Pm-S-Ba

showed a difference between the 2 groups; this may be due to a combination of many factors, such as decreased maxillary length and cranial base angle in JD patients. The percentage of posterior face height to total anterior face height is not significantly decreased in JD patients. In addition, the percentage of the lower anterior face height to the total anterior face height was not significantly decreased. This may be due to the decrease of all linear measurements in JD patients. Most of our results are in agreement with the results reported by El-Bialy, et. al ${ }^{17}$ This may be due to similar etiological factors of the JD in the middle east, however, this speculation would require further investigation to validate it.

\section{Summary and Conclusion}

This study investigated the effects of juvenile diabetes mellitus on craniofacial morphology and growth as well as on skeletal maturation. The sample consisted of 30 male patients with ages between 14 and 16 years. All of these patients were diagnosed with diabetes not less than 5 years before the study, and none displayed any other diseases that could affect general body development or craniofacial morphology.

The control group consisted of 20 normal males in the same age range (14 to 16 years) without a display of any disease that could affect general body development or craniofacial morphology paired with the JD patients. Lateral cephalometric radiographs were taken for each subject. JD patients were shown to be underdeveloped in most of the parameters when compared with the control group. Although further studies are needed to confirm the findings of this study, 
it appears that craniofacial morphology and skeletal maturation are affected by diabetes mellitus, especially before and during circumpubertal growth spurts. These findings should be considered when craniofacial orthopedic problems are diagnosed and treated injuvenile diabetic patients.

\section{REFERENCES}

1. Lebovitz H. Etiology and pathogenesis of Diabetes Mellitus: Pediatr Clin North Am. 1984;521-30.

2. Sperling, M. Diabetes mellitus In Nelson, Textbook of Pediatrics Ed. Behrman R.E., $13^{\text {th }}$ Ed.. Philadelphia, London, Toronto: 1987;1248-62.

3. National Diabetes Data Group. Classification and diagnosis of diabetes mellitus and other categories of glucose intolerance: Diabetes 1979;1039-57.

4. Duncan, G. Diseases of metabolism. $4^{\text {th }}$ Ed. Sunders $1961 ; 911$.

5. Rosenbloom,AL, Silverstein, JH, Riley, WJ. Joint contracture in childhood diabetes indicates high risk for vasculopathy: Pediatr. Adolesc Endocrinol. 1981;143-9.

6. Calnen, $\mathrm{M}$ and Pekham, CS. Incidence of insulin dependent diabetes in the first sixteen years of life: Lancet. 1977;589-90.

7. Fleeger, FM, Roger KD, Drash, A, Rosenbloom, AL, Travis, LB, and Court, JM. Age, sex and season of onset of juvenile diabetes in different geographic areas: Pediatrics. 1979;374-79.

8. Warsy AS, el-Hazmi MA.:Diabetes mellitus, hypertension and obesity--common multifactorial disorders in Saudis. East Mediterr Health J. 1999 Nov;5(6):1236-42.

9. Ali, OF, Hanafy, FZ. Sociomedical study on epidemiology of juvenile onset diabetes mellitus in Mansura school age children under 15 years. Egypt. J. Comm. 1986;131-50.

10. Pastan, RS, and Cohen, AS. The rheumatologic manifestations of diabetes mellitus. Med. Clin. North. Am. 1978;829-39.

11. Schneider SL; Kohn RR . Glucosylation of human collagen in aging and diabetes mellitus. J Clin Invest 1980;1179-81.

12. Rosenbloom, AL. Limited joint mobility in diabetes mellitus of childhood: Natural history and relationship to growth impairment. J. Pediatr. 1982;874-78.

13. Bogan IK. Hands and wrists of diabetic child. Am. J Dis. Child. 1941;815-65. 
14. Goldstein, S, Littlefield, JW, Soldner, JS. Diabetes mellitus and bone diminished plating efficiency of cultured human fibroblasts. Post. Grad. Acad. Sci. USA. $1969 ; 155-160$.

15. McNair, P. Development of bone mineral loss in insulin treated diabetes: a 1 years follow-up in sixty patients. Europ. J. Clin. Indoc. 1980;55-59.

16. Roe TF, Mora, S, Costen, G, Kaufman, F, Carlson, M, and Gilsanz, V. Vertebral bone density in insulin-dependent diabetic children. Metabolism. 1991;967-71.

17. El-Bialy T, Aboul-Azm SF, El-Sakhawy M.: Study of craniofacial morphology and skeletal maturation in juvenile diabetics (Type I). Am J Orthod Dentofacial Orthop 2000 Aug; 118(2):189-95

18. Fishman, LS. Radiographic evaluation of skeletal maturation. Angle Orthod. 1982;88-112.

19. Chew, FS. Radiologic manifestations in the musculoskeletal system of miscellaneous endocrine disorders. Radiol. Clin. North. Am. 1991;34-47.

20. Craig, JO. Growth as a measurement of control in the management of diabetic children. Post. Grad. Med J. Suppl. 1970;607.

21. Sterky, G. Growth pattern in juvenile diabetes. Acta Ped. Scand. Sup. 1967;17788.

22. Broadbent, BH. Bolton standards of dentofacial developmental growth. Mosby, Saint Louis. 1975;1-2.

23. Moore, RN, Moyer, BA, and DuBois, LM. Skeletal maturation and craniofacial growth. Am. J. Orthod. 1990:98;33-40.

24. Kerr, WJ. A longitudinal cephalometric study of dentofacial growth from 5 to 15 years. Brit. J. Orthod. 1979:6;115-121.

25. Pileski, RC, Woodside, DG, and James, GA. Relationship of the ulnar sesamoid bone and maximum mandibular growth velocity. Angle. Orthod. 1972:42;162-70. 